\title{
Use of high resolution computed tomography of the lungs in patients with rheumatoid arthritis
}

Bernard Cortet, René-Marc Flipo, Martine Rémy-Jardin, Pascal Coquerelle, Bernard Duquesnoy, Jacques Rémy, Bernard Delcambre

\begin{abstract}
Objective-To assess the usefulness of high resolution computed tomography (HRCT) of the lungs in patients with rheumatoid arthritis (RA) with and without respiratory symptoms.

Patients and methods-Eighty eight RA patients with a mean duration of disease 12 (SD 8) years were evaluated. Eleven patients were excluded because of previous exposure to silica. The 77 remaining patients formed two groups according to the absence (group $I, n=38$ ) or the presence (group II, $n=39$ ) of chronic respiratory symptoms. A control group consisted of 51 non-smoking, healthy patients.
\end{abstract}

Results-The most frequent abnormalities observed in the 77 RA patients were bronchiectasis or bronchiolectasis $(n=23$, $30 \%)$, pulmonary nodules $(n=17,22 \%)$, subpleural micronodules or pseudoplaques ( $n=13,17 \%)$, ground glass opacities $(n=11,14 \%)$, and honeycombing $(n=8$, $10 \%$ ). Bronchiectasis or bronchiolectasis $(p=0 \cdot 012)$, rounded opacities $(p=0 \cdot 016)$, ground glass attenuation $(p=0 \cdot 004)$, and honeycombing $(p=0.002)$ were found more often in RA group II (with respiratory symptoms) than in group I (no respiratory symptoms). Non-linear septal opacities were more frequent in group $I$ than in the control group, but other HRCT findings did not differ statistically significantly between group I and the control group. Conclusion-Bronchiectasis may be a characteristic lung change in $R A$ patients. Abnormalities on HRCT are less frequently observed in the absence of respiratory symptoms than in the presence of such symptoms (29\% versus $69 \%)$.

(Ann Rheum Dis 1995; 54: 815-819)

Rheumatoid arthritis (RA) is a common chronic inflammatory disease affecting about $1 \%$ of the white population. Extra-articular manifestations may involve, in particular, the skin (rheumatoid nodules), eyes, heart, and lungs. Since the first description of lung disease associated with RA by Ellman and Ball in $1948,{ }^{1}$ several forms of pleuropulmonary disease have been established in RA: pleural effusion/ pleuritis, rheumatoid lung nodules, Caplan's syndrome, fibrosing alveolitis, lymphoid hyperplasia with germinal centres, pulmonary hypertension, constrictive bronchiolitis, and bronchiectasis. ${ }^{2}$ The prevalence of lung manifestations in RA is not known with accuracy, and varies according to the method of diagnosis. The chest radiograph, for example, may be abnormal in $2-5 \%$ of patients with RA, ${ }^{2}$ but plain chest radiography is not sensitive enough for the diagnosis of lung involvement. High resolution computed tomography (HRCT) is a non-invasive method of assessing interstitial lung disease (ILD), in particular, which has recently been shown to be useful in systemic sclerosis. ${ }^{34}$ The use of HRCT in RA is poorly documented, but the technique appears to be useful when there is suspected clinical and radiological ILD. ${ }^{56}$ The aim of the present study was to assess the usefulness of HRCT in RA patients with and without respiratory symptoms, compared with a control group of non-smoking volunteers.

\section{Patients and methods}

PATIENTS

Eighty eight patients fulfilling the revised criteria for RA of the American Rheumatism Association were reviewed. ${ }^{7}$ All had undergone HRCT examination of the thorax between 1987 and 1993. Among them, 11 patients who had been exposed to silica were excluded because of possible confusion between rheumatoid and pneumoconiotic lung lesions. HRCT had been performed in the remaining 77 patients because of suspicion of associated pulmonary disease, on the grounds of pulmonary symptoms or systematic evaluation of lung changes. In the latter case, the patients were selected consecutively from one department of rheumatology, the systematic evaluation of their lung changes was approved by the Hospital Ethics Committee, and all patients gave written informed consent to participate. The 77 patients comprised 51 women and 26 men, mean age 57 (SD 10) years (range 36-79). Seventy one were non-smokers who had never smoked and six were current smokers, mean cigarette consumption 24 (20) packs/year (range 1-60 packs). The mean duration of RA at the time of chest HRCT was 12 (8) years. Subcutaneous rheumatoid nodules were noted in 17 patients $(22 \%)$ and rheumatoid factor in $44(57 \%)$. Nine patients $(12 \%)$ were suffering from Sjögren's syndrome (presence of sicca syndrome and positive labial salivary gland biopsy (focus score $>1$, stage III or IV on Chisolm's classification $\left.{ }^{8}\right)$ ). Functional capacity was evaluated by Steinbrocker classification: ${ }^{9}$ 12 were class I, 28 class II, and 37 class III. At the time of the HRCT examination, 63 of \\ Accepted for publication
}


the 77 patients were receiving corticosteroids or second line drugs: glucocorticoids $(n=49)$; methotrexate $(n=24)$; tiopronine $(n=10)$; sodium aurothiomalate $(n=7)$; sulphasalazine $(n=7)$; hydroxychloroquine $(n=1)$. The patients were allocated to two groups according to the absence (group I, $\mathrm{n}=38$ ) or presence (group II, $\mathrm{n}=39$ ) of respiratory symptoms such as cough (in the morning or all day), sputum production (in the morning or all day), or dyspnoea.

A control group comprised 51 healthy subjects who had never smoked and who were previously enrolled in a prospective study assessing HRCT of the lungs in healthy adult volunteers. ${ }^{10}$ There were 34 women and 17 men (mean age 33 (8) years), all urban dwellers and recruited from workers in our hospital. None had a past history of lung disease and, in particular, none had previously undergone chest surgery or suffered from a respiratory illness such as bronchiolitis of any origin in previous years or in infancy. Some control patients had respiratory symptoms: cough ( $n=4$; morning, $n=3$; all day, $n=1)$, sputum production in the morning $(n=2)$, and dyspnoea occurring after strenuous activity such as climbing three flights of stairs, heavy housework, or walking more than one mile on level ground $(n=8)$.

\section{METHODS}

HRCT of the thorax was performed with either an Elscint 2400 (Hackensak, NJ) or a Siemens Somatom Plus (Erlangen, Germany). Serial slices were taken through the chest, each $1 \mathrm{~mm}$ in width and $10 \mathrm{~mm}$ apart. Technical factors were $130 \mathrm{kV}$ and $420 \mathrm{~mA}$ (Elscint CT unit) or $137 \mathrm{kV}$ and $255 \mathrm{~mA}$ (Siemens CT unit). Images were reconstructed using a high spatial frequency algorithm for parenchymal analysis and a standard algorithm for mediastinal evaluation. HRCT studies were performed at suspended end inspiratory volume with one second (Siemens) or two seconds (Elscint) scan time with patients in the supine position. In cases of limited joint mobility, especially involving the shoulders, HRCT scan was performed with the patient's arms positioned alongside the body, without any effect on the image quality. The HRCT examinations were interpreted by two radiologists blind to the clinical history, who reached a concensus

High resolution computed tomography (HRCT) findings in patients with rheumatoid arthritis $(R A)$ without (group I) and with (group II) respiratory symptoms

\begin{tabular}{lllc}
\hline HRCT finding & $\begin{array}{l}\text { Group I } \\
(n=38)\end{array}$ & $\begin{array}{l}\text { Group II } \\
(n=39)\end{array}$ & $\begin{array}{c}\text { Control group } \\
(n=51)\end{array}$ \\
\hline Bronchial wall thickening & $2(5)$ & $11(28)$ & $9(18)$ \\
Bronchiectasis or bronchiolectasis & $3(8)$ & $20(51)^{\star}$ & 0 \\
Rounded opacities & $4(11)$ & $13(33)^{\star}$ & $11(22)$ \\
Septal lines & 0 & 0 & $4(8)$ \\
Non-septal linear opacities & $5(13)$ & $9(23)$ & $0^{\star}$ \\
Ground glass attenuation & $1(3)$ & $10(26)^{\star \star}$ & 0 \\
Honeycombing & 0 & $8(23)^{\star \star}$ & 0 \\
Dependant areas of attenuation & $1(3)$ & $4(10)$ & $6(12)$ \\
Distortion & $1(3)$ & $4(10)$ & 0 \\
Emphysema & $2(5)$ & $2(5)$ & 0 \\
Enlarged lymph nodes & $2(5)$ & $5(13)$ & 0 \\
Enlarged pulmonary arteries & 0 & $1(3)$ & 0 \\
Pleural abnormalities & $3(8)$ & $9(23)$ & 0
\end{tabular}

$\mathrm{n}=$ Number of patients with HRCT findings. Number in parentheses are percentage. ${ }^{\star} \mathrm{p}<0.05$ ${ }_{\star \star \star} \mathrm{p}<0.01$ compared with group $\mathrm{I}$. decision. Multiple radiological criteria for the diagnosis of lung involvement were assessed, including site and severity. The major abnormalities screened for were: rounded opacities (parenchymal micronodules $=$ rounded lesions less than $3 \mathrm{~mm}$ in diameter; nodules = rounded lesions greater than $3 \mathrm{~mm}$ in diameter; subpleural micronodules $=$ areas of hyperattenuation less than $3 \mathrm{~mm}$ in diameter); ground glass attenuation (bronchi and vessels visible); dependent areas of attenuation (bronchial walls and vessels obscured); septal lines and non-septal lines; honeycombing (areas of cystic spaces with thickened walls); bronchiectasis (abnormal visualisation of proximal airways); bronchiolectasis (abnormal visualisation of airways in peripheral locations: dilated bronchiolar division visualised along their length when horizontal, or peripheral signet ring signs when coursing in a vertical direction; architectural distortion; emphysema characterised by areas of decreased attenuation and disruption of the vascular pattern.

Statistical comparisons between the different groups of patients were made using the $\chi^{2}$ test with Yates' correction if necessary. Correlation coefficients were calculated by linear regression analysis.

\section{Results}

The table summarises the results.

HRCT IN RA PATIENTS

HRCT was abnormal in $38(49 \%)$ of the 77 RA patients-11 from group I (29\%) (no respiratory symptoms) and 27 from group II (69\%) (with respiratory symptoms) $(\mathrm{p}<0.001)$.

The most frequent abnormality on HRCT of the lungs was bronchiectasis or bronchiolectasis (figure), observed in 23 patients (30\%)-three patients in group $I(8 \%)$, and 20 patients in group II $(51 \%)(p=0.012)$-and consisted of honeycombing in seven of them. Among the 16 with bronchiectasis or bronchiolectasis but no honeycombing, 14 patients were non-smokers who had never smoked. Specific evaluation of these 14 non-smoking patients showed bronchiectasis or bronchiolectasis associated with diffuse bronchial thickening in all but one; in four, airway changes were the sole abnormality on HRCT examination, and in 10 the airway changes were associated with parenchymal micronodules $(n=6)$, dependent areas of attenuation $(n=4)$, subpleural micronodules or pseudoplaques $(n=4)$ and emphysema $(n=2)$.

Rounded opacities were observed in 17 patients $(22 \%)$. Three types were identified according to their size and location: parenchymal micronodules $(n=6)$, nodules $(n=3)$, and subpleural micronodules or pseudoplaques $(n=13)$.

In the absence of respiratory symptoms (group I), rounded opacities were observed in four patients $(11 \%)$, peripheral parenchymal micronodules in two, and subpleural micronodules in two. In group II (with respiratory symptoms), 13 patients (33\%) had rounded opacities: parenchymal micronodules in four, 


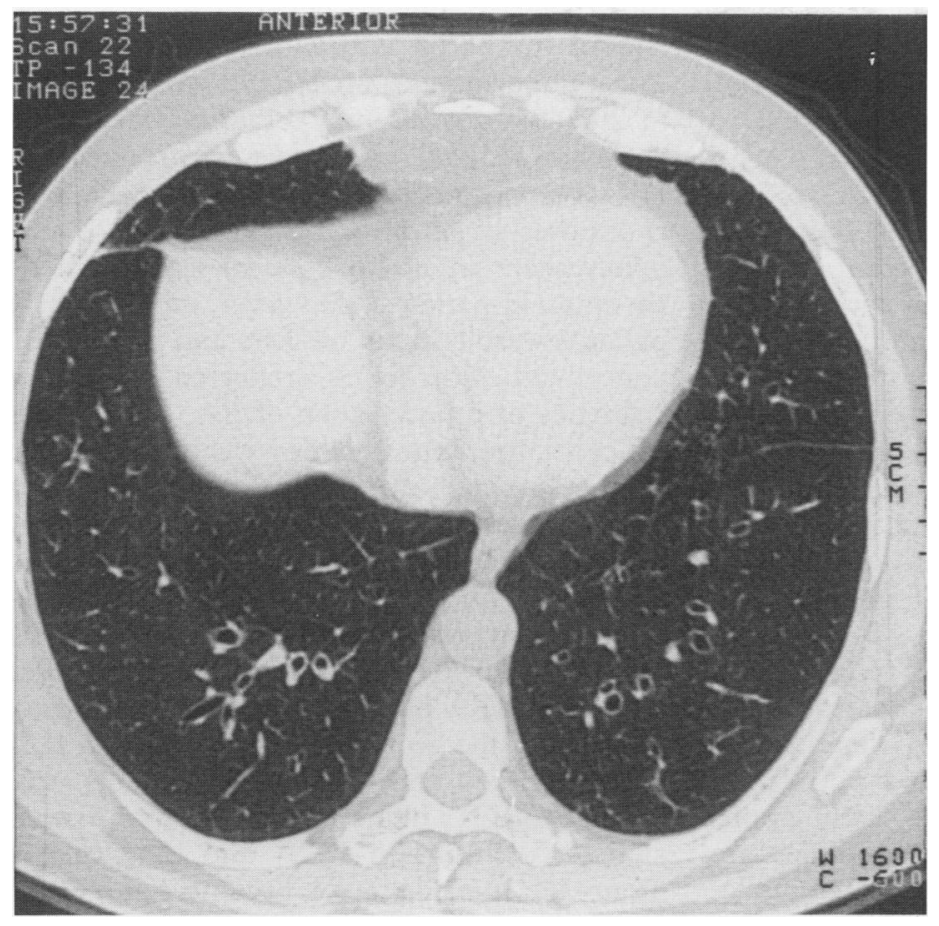

Bronchiectasis: cylindral dilatation of peripheral bronchi in the lower lobes and in the lingula, with mild bronchial wall thickening in the posterior segment of the right lower lobe.

parenchymal nodules in three, and subpleural micronodules in 11 . This difference between the groups was statistically significant $(p=0 \cdot 016)$.

Non-septal linear opacities were observed in 14 patients (18\%) and were always located in peripheral lung tissue. Such abnormalities were the sole HRCT findings in six patients and were associated with honeycombing in eight; they were exclusively or predominantly in the lower lung zones in all cases.

Five patients $(13 \%)$ had non-septal linear opacities in the absence of respiratory symptoms (group I) and nine (23\%) with this feature had respiratory symptoms (group II) (difference between groups not significant).

Ground glass attenuation representing early active alveolitis was found in 11 patients (14\%) -one in group I (3\%) and 10 in group II $(10 \%)(p=0 \cdot 004)$. It was usually bilateral and symmetrical.

Honeycombing highly suggestive of pulmonary fibrosis was detected in eight patients $(10 \%)$ and comprised a microcystic pattern $(n=5)$, or a combination of microcystic and macrocystic honeycombing $(n=3)$. Honeycombing was always bilateral and asymmetrical, and was associated with bronchiectasis or bronchiolectasis in seven patients, ground glass attenuation in seven, non-septal linear opacities in all cases, and pleural thickening or pleural effusion in five. Evidence of honeycombing was always associated with presence of respiratory symptoms (significant difference between groups $(p=0.002))$.

The presence of respiratory symptoms (cough, dyspnoea or sputum production) was significantly correlated with the following HRCT findings: rounded opacities $(p=0.016)$, ground glass attenuation $(p=0.004)$, honeycombing $(p=0.002)$, and bronchiectasis or bronchiolectasis $(p<0.001)$.
HRCT IN THE CONTROL GROUP

Abnormalities were evident on HRCT in 22 control patients (43\%). The most frequent abnormality was subpleural micronodules ( $n=11,22 \%$ ); others were: bronchial wall thickening without any other bronchial abnormality ( $n=9,18 \%)$, dependent areas of attenuation $(n=6,12 \%)$ and septal lines $(\mathrm{n}=4,8 \%)$. A significant correlation between each respiratory symptom and HRCT findings was found only for bronchial wall thickening $(p \leqslant 0.009)$.

\section{COMPARISON OF HRCT IN RA PATIENTS AND} CONTROLS

Bronchiectasis or bronchiolectasis $(p<0.001)$, septal lines $(p=0.05)$, non-septal linear opacities $(p<0.01)$, ground glass attenuation $(p<0.01)$, honeycombing $(p<0.05)$, and pleural abnormalities $(p<0.01)$ occurred more frequently in RA patients than in the control group. Non-septal linear opacities was the sole abnormality observed more frequently on HRCT in RA patients without respiratory symptoms (group I) than in control patients $(p<0.01)$.

\section{Discussion}

The most frequent abnormality depicted on HCRT in our study was bronchiectasis or bronchiolectasis-found in 23 patients (30\%). While seven of these patients had lung changes (honeycombing) considered as indirect signs of pulmonary fibrosis, the remaining $16 \mathrm{RA}$ patients with abnormalities in the absence of features of lung fibrosis included 14 nonsmokers who had never smoked. This strongly suggests that neither smoking nor an increased susceptibility to its effects were the main causes for the development of bronchiectasis or bronchiolectasis. In addition, we failed to observe bronchiectasis/bronchiolectasis in our control group of non-smokers, among whom bronchial abnormalities consisted solely of bronchial wall thickening which could be explained by occupational exposure to pollutants or other environmental factors (urban dwellers). An association between bronchiectasis and RA is well known, ${ }^{11}$ but it remains unclear if bronchiectasis occurs in severe RA and if it is accompanied by other extra-articular manifestations. ${ }^{12}{ }^{13} \mathrm{McD}$ onagh, et al, using HCRT, found bronchiectasis in four of 20 RA patients (20\%), but none was suffering from clinical symptoms of respiratory disease. ${ }^{6}$ In the same study, the authors observed bronchiectasis in $30 \%$ of the patients with interstitial lung disease. On the basis of pulmonary function tests, it has been shown that airway disease may be the commonest form of RA lung involvement; ${ }^{14}$ in other RA patients, low values of forced expiratory volume in one second $\left(\mathrm{FEV}_{1}\right)$, forced vital capacity (FVC), $\mathrm{FEV}_{1} / \mathrm{FVC}$, and a significant high prevalence of bronchial reactivity to inhaled methacholine have been reported. ${ }^{15}$ The most common explanation is that bronchiectasis in RA patients results from frequent respiratory tract infections. ${ }^{16}$ The 
predominant location of bronchiectasis or bronchiolectasis in the lower zones of the lungs supports this hypothesis. In addition, alpha-1 antitrypsin deficiency has been suggested to increase the risk of lung involvement in RA. ${ }^{17}$

Rounded opacities, found in $22 \%$ of our patients, were the second most frequently observed abnormality. Their location and size were consistent with the characteristic subpleural location of rheumatoid nodules. ${ }^{18}$ Pulmonary nodules are usually symptomless, or contribute little to pulmonary dysfunction; however, they can grow in size and tend to rupture into the pleura causing pneumothorax, hydropneumothorax, or even pyopneumothorax, as observed in one of our patients. ${ }^{19} 20$ The frequency of parenchymal nodules in our patients (three in $77-4 \%$ ) was lower than that reported from pathological studies ${ }^{18}$ and on previous HRCT scan studies, ${ }^{6}$ even though HRCT is able to demonstrate lung nodules in the rheumatoid population more frequently than plain radiographs (fewer than $1 \%$ of rheumatoid nodules are estimated to be radiographically detectable ${ }^{21}$ ). We observed subpleural micronodules in $17 \%$ of our RA patients; although subpleural micronodules were identified in $22 \%$ of our control group, several CT findings suggest their possible relationship with rheumatoid lung disease. Subpleural lesions in RA patients are observed in the upper, mid and lower lung zones, whereas in healthy adults subpleural micronodules are exclusively located in the upper part of the lungs. ${ }^{10}$

Ground glass opacification representing an early stage of active alveolitis was the fourth most frequent HCRT abnormality we detected $(14 \%)$. Discovery of such an abnormality on HRCT could indicate early aggressive treatment to prevent irreversible fibrosis.

Honeycombing was present in eight patients $(10 \%)$, always bilateral and asymmetrical, with a predominant peripheral and inferior distribution. These morphological features are in agreement with those previously reported in the literature, ${ }^{22}{ }^{23}$ and are indistinguishable from pulmonary fibrosis caused by other connective tissue diseases. ${ }^{24}$ Although interstitial lung disease is a well known extraarticular manifestation of RA, its prevalence is closely related to the diagnostic tool used for its recognition. Pathological studies have shown interstitial lung disease in up to $80 \%$ of patients, ${ }^{1825}$ whereas interstitial lesions are radiographically identified in fewer than $5 \% .{ }^{26}$ Although the aim of this study was not to compare chest radiography with lung HRCT, we are able to confirm the lack of sensitivity of plain chest radiography, as $90 \%$ of our patients suffering from lung fibrosis had a normal chest radiograph.

Emphysema was seen in 5\% of our RA patients, whereas McDonagh et $a l^{6}$ found it in $20 \%$. Tobacco smoking may explain this difference, as $80 \%$ of their patients were current smokers, whereas $90 \%$ of those in our study were non-smokers and had never smoked. The prevalence of HRCT findings according to the absence or presence of respiratory symp- toms likewise differed between these studies. Among our 38 patients with respiratory symptoms, abnormalities on HRCT were observed in $29(69 \%)$, while only $11(29 \%)$ of our 39 patients without respiratory symptoms had HRCT abnormalities. In contrast, McDonagh et al observed on HRCT a prevalence of lung involvement in up to $75 \%$ of asymptomatic rheumatoid patients. ${ }^{6}$ However, most of these patients were current smokers and the HRCT abnormalities probably reflected a greater influence of tobacco than of RA. The significance of the abnormalities we demonstrated in the absence of respiratory symptoms is unknown, as we did not perform pulmonary function tests. While McDonagh's group did not find any significant difference on pulmonary function testing between RA patients with clinical evidence of interstitial lung disease and control RA patients, when the pulmonary function of the group of 20 patients with ILD was compared with that of 10 patients with normal HRCT, or nodules or pleural disease alone, reductions in $\mathrm{FEV}_{1}$ and lung carbon monoxide transfer factor became significant. ${ }^{6}$ Apart from the non-septal linear opacities which were more frequent in our RA patients without respiratory symptoms (group I) than in control patients, the frequency of HRCT abnormalities was similar in these two groups. However, the fact that these groups were not age matched should be taken into account when interpreting this result. Finally, in the absence of respiratory symptoms, slight ground glass opacification representative of active alveolitis was demonstrated in only one patient, and the honeycombing considered to indicate lung fibrosis was not observed.

In summary, HRCT is a useful diagnostic tool in the assessment of lung involvement in patients with respiratory symptoms and offers information not readily obtained from plain chest radiography. The significance of the several abnormalities which were observed in the absence of respiratory symptoms remains unclear and requires subsequent longitudinal studies.

1 Ellman P, Ball R E. Rheumatoid disease with joint and pulmonary manifestations. BMF 1948; 2: 816-20.

2 Kelly C A. Rheumatoid arthritis: classical lung disease. In: Kelly C A, ed. Lung disease in rheumatic disorders. London: Baillière Tindall, 1993; 1-17.

3 Hansell D M, Kerr I H. The role of high resolution computed tomography in the diagnosis of interstitial lung disease. Thorax 1991; 46: 77-85.

4 Harrison N K, Glanville N R, Strickland B, et al. Pulmonary involvement in systemic sclerosis: the detection of early changes by thin section $\mathrm{CT}$, bronchoalveolar lavage and $99 \mathrm{~m}$ Tc-DTPA clearance. Respir Med 1989; 83: 403-14.

5 Fewins H E, McGowan I, Whitehouse G H, Williams J, Mallya R. High definition computed tomography in rheumatoid arthritis associated pulmonary disease. $\mathrm{Br} \mathcal{F}$ Rheumatol 1991; 30: 214-6.

6 McDonagh J, Greaves M, Wright A R, Heycock C Owen J P, Kelly C. High resolution computed tomography of the lungs in patients with rheumatoid arthritis and interstitial lung disease. $B r f \mathcal{F}$ Reumatol 1994; 33 118-22.

7 Arnett F C, Edworthy S M, Bloch D A, et al. The American Rheumatism Association 1987 revised criteria for the classification of rheumatoid arthritis. Arthritis Rheum 1988; 31: 315-24.

8 Chisolm D M, Mason D K. Labial salivary gland biopsy in Sjögren's disease. $\mathcal{F}$ Clin Pathol 1968; 21: 656-60.

9 Steinbrocker O, Trager C H, Batterman R C. Therapeutic criteria in rheumatoid arthritis. $\mathscr{f} A M A$ 1949; 37: 393-406. 
10 Remy-Jardin M, Remy J, Boulenguez C, Sobaszek A, Edme J-L, Furon D. Morphologic effects of cigarette smoking on airways and pulmonary parenchyma in healthy adult volunteers: CT evaluation and correlation with pulmonary function tests. Radiology 1993; 186: with pulm.

11 Solanki T, Neville E. Bronchiectasis and rheumatoid disease: is there an association? Br $\mathcal{f}$ Rheumatol 1992; 31: 691-3.

12 McMahon M J, Swinson D R, Shettar S, et al. Bronchiectasis and rheumatoid arthritis: a clinical study. Ann Rheum Dis 1993; 52: 776-9.

13 Shadick N A, Fanta C H, Weinblatt M E, Odonnell W, Coblyn J S. Bronchiectasis. A late feature of severe rheumatoid arthritis. Medicine (Baltimore) 1994; 73: 161-70.

14 Geddes D, Webley H, Emerson P. Airway obstruction in rheumatoid arthritis. Ann Rheum Dis 1979; 38: $222-5$.

15 Hassan W U, Keaney N P, Holland C D, Kelly C A. Bronchial reactivity and airflow obstruction in Bronchial reactivity and airflow obstruction

16 Bamji A, Cooke N. Rheumatoid arthritis and chronic bronchial suppuration. Scand $\mathcal{f}$ Rheumatol 1985; 14: $15-21$

17 Geddes D M, Webley M, Turton C W, et al. Alpha 1 antitrypsin phenotypes in fibrosing alveolitis and rheumatoid arthritis. Lancet $1977 ; 2$ : 1049-50.
18 Yousem S A, Colby T V, Carrington C B. Lung biopsy in rheumatoid arthritis. Am Rev Respir Dis 1985; 131: 770-7.

19 Portner M M, Gracie W A. Rheumatoid lung disease with cavitary nodules, pneumothorax and eosinophilia. $N$ Engl f Med 1966; 275: 697-700

20 McConnoche K, O'Sullivan M, Khalh J F, Purchard M H, Gibbs A R. Aspergillus colonization of pulmonary rheumatoid nodule. Respir Med 1989; 83: 157-60.

21 Walker W C, Wright V. Pulmonary lesions and rheumatoid arthritis. Medicine (Baltimore) 1968; 47: 501-20.

22 Steinberg D L, Webb R W. CT appearances of rheumatoid lung disease. $f$ Comput Assist Tomogr 1984; 8: 881-4.

23 Fuji M, Adachi S, Shimizu T, Hirota S, Sako M, Kono M. Interstitial lung disease in rheumatoid arthritis: assessment with high-resolution computed tomography. ₹ Thorac Imaging 1993; 8: 54-62.

24 Remy-Jardin M, Remy J, Wallaert B, Bataille D, Hatron $P$ Y. Pulmonary involvement in progressive systemic sclerosis. Sequential evaluation with CT, pulmonary function tests and bronchoalveolar lavage. Radiology 1993; 188: 499-506.

25 Cervantez-Perez P, Toro-Perez A H, Rodriguez-Jurado P. Pulmonary involvement in rheumatoid arthritis. $\mathfrak{F} A M A$ 1980; 243: 1715-7

26 Walker W C, Wright V. Diffuse interstitial pulmonary fibrosis and rheumatoid arthritis. Ann Rheum Dis 1969; 28: $252-9$. 